\title{
Kinetic Modeling of Dissolution and Crystallization of Slurries with Attenuated Total Reflectance UV-Visible Absorbance and Near- Infrared Reflectance Measurements
}

\author{
Chun H. Hsieh, ${ }^{\dagger}$ Julien Billeter, $^{\dagger}$ Mary Ellen P. McNally, $^{\ddagger}$ Ronald M. Hoffman, ${ }^{\ddagger}$ and Paul J. Gemperline* ${ }^{\dagger}$ \\ ${ }^{\dagger}$ Department of Chemistry, East Carolina University, Greenville, North Carolina, United States \\ ${ }^{\ddagger}$ Crop Protection Products and Engineering Technologies, Stine Haskell Research Center, E.I. DuPont de Nemours and Company, \\ Incorporated, Newark, Delaware, United States
}

ABSTRACT: Slurries are often used in chemical and pharmaceutical manufacturing processes but present challenging online measurement and monitoring problems. In this paper, a novel multivariate kinetic modeling application is described that provides calibration-free estimates of timeresolved profiles of the solid and dissolved fractions of a substance in a model slurry system. The kinetic model of this system achieved data fusion of time-resolved spectroscopic measurements from two different kinds of fiber-optic probes. Attenuated total reflectance UV-vis (ATR UV-vis) and diffuse reflectance near-infrared (NIR) spectra were measured

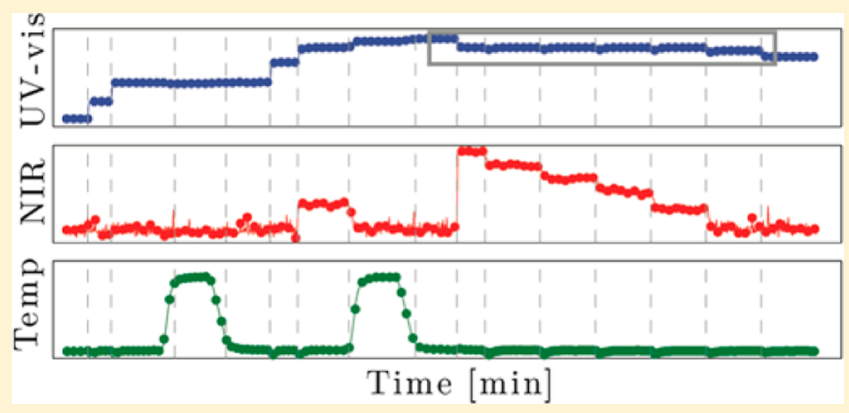
simultaneously in a small-scale semibatch reactor. A simplified comprehensive kinetic model was then fitted to the time-resolved spectroscopic data to determine the kinetics of crystallization and the kinetics of dissolution for online monitoring and quality control purposes. The parameters estimated in the model included dissolution and crystal growth rate constants, as well as the dissolution rate order. The model accurately estimated the degree of supersaturation as a function of time during conditions when crystallization took place and accurately estimated the degree of undersaturation during conditions when dissolution took place.

S ignificant progress in the area of multivariate batch process monitoring, modeling, and control has been made over the last 2 decades; ${ }^{1}$ however, strategies for monitoring and modeling of slurries have not been widely reported, despite the fact that slurries are often used in chemical and pharmaceutical manufacturing processes. Many of the early developments in process analysis can be attributed to groundbreaking work of Nomikos and MacGregor ${ }^{2,3}$ and Wold and co-workers. $^{4-6}$ These efforts were largely focused on the use of principal component analysis (PCA) and partial least-squares (PLS) to develop multivariate statistical process control (MSPC) models for characterization of process operating conditions and thereafter the definition of normal operating conditions for the production of batches fulfilling the desired specifications. ${ }^{1}$ These models were then used to monitor future batches, product quality, and yield, as well as detect faults and diagnose process deviations.

An alternative to this approach called multivariate kinetic modeling has also seen significant development over the last 2 decades. In these approaches, first-principles physical models are fitted directly to multivariate spectroscopic measurements where, typically, the adjustable model parameters are rate constants. $^{7-10}$ Recently, kinetic model fitting methods were extended to achieve fusion of calorimetric measurements of univariate nature with multivariate spectroscopic measurements, ${ }^{11-14}$ extended to incorporate chemical equilibria, ${ }^{15,16}$ used for estimation of additional parameters such as activation energies and reaction enthalpies, ${ }^{17,18}$ and for fitting of extents of reaction in gas-liquid systems. ${ }^{19}$ These modeling approaches offer some advantages and some drawbacks compared to empirical multivariate statistical process control models. One significant advantage of multivariate kinetic fitting is that these methods are "calibration-free". ${ }^{18}$ By fitting models directly to time-resolved spectra, development of independent spectroscopic calibration models for estimating concentration profiles as a function of time becomes unnecessary. Prerun batches can be used to estimate model parameters by the use of global models ${ }^{15}$ (one reaction scheme fit to multiple batches). Also, it has been demonstrated that models can be scaled up ${ }^{20}$ or extended to new operating conditions. ${ }^{21}$ For real-time batches, the model parameters can be updated to forecast end points and estimate product yields and product quality. ${ }^{18,22}$ While postulating and identifying the correct physical model is more challenging in multivariate kinetic modeling approaches, this difficulty may be offset by the benefits of increased process insight as well as a significant reduction in the requirement for large libraries of historical batch data normally required in MSPC approaches.

Received: September 23, 2012

Accepted: April 9, 2013

Published: April 9, 2013 
The use of slurries in industrial processes involves dissolution and crystallization; therefore, a useful kinetic model of such a process must include features that account for this added complexity. The development of population balance equations (PBEs) for nucleation and crystal growth can be traced to the ground-breaking work of Randolph and Larson. ${ }^{23}$ Early work focused on understanding of fluid mechanics, nucleation kinetics, growth kinetics, and crystallization process performance. $^{24,25}$ Significant progress has been made on the use of PBEs for modeling crystallization processes, often by use of calorimetric data and off-line solubility determinations. ${ }^{26-32}$ Monitoring of solubility and supersaturation by the use of in situ spectroscopic methods has also been reported, ${ }^{33}$ and the fitting of PBE-based models to such solubility and supersaturation curves derived from calibrated in situ spectroscopic measurements has also been reported. ${ }^{34-39}$

The development of dissolution models dates back more than 100 years with the development of the Noyes-Whitney equation. ${ }^{40}$ Some recent work includes the development of a lattice-Boltzman model, ${ }^{41}$ which under appropriate assumptions can be used to simulate complex reactive flows such as in porous media. Most recently, Gao reported the integration of solid-liquid interface kinetics and mass transport kinetics to treat special conditions such as ionization equilibria and supersaturation. $^{42}$

Recent progress has also been made on the fitting of kinetic models to heterogeneous systems involving crystallization and dissolution processes. Cornel and co-workers were the first to report this kind of application of multivariate kinetic fitting for modeling polymorphic transformations. ${ }^{43,44}$ In their approach, a PBE-based model was fitted directly to time-resolved in situ Raman spectra ${ }^{43}$ or attenuated total reflectance Fourier transform infrared (ATR-FT-IR) spectra, ${ }^{44}$ thereby avoiding the need to build and maintain independent calibration models for estimating concentration profiles as a function of time.

In this paper, we report the combination of a traditional dissolution model and a crystallization model and demonstrate the combined approach to slurries involving dissolution and crystallization processes by fusing two different kinds of spectroscopic measurements, UV-vis ATR measurements with near-infrared (NIR) reflectance measurements and reagent flow-in conditions into a semiempirical model. The ATR UVvis measurements inform the model fitting process about the concentration of the solute in the solution phase, and the NIR reflectance measurements inform the model about the presence and amount of solid material in the crystalline phase. A small laboratory-scale semibatch reactor was used to produce data for kinetic fitting and provided a means of precise control of experimental conditions. The model is used to accurately estimate time-resolved concentration profiles, the degree of supersaturation during conditions when crystallization takes place, and the degree of undersaturation during conditions when dissolution takes place.

\section{EXPERIMENTAL SECTION}

A series of four batch experiments were conducted using a 50 $\mathrm{mL}$ reactor at East Carolina University in Greenville, NC. In situ ATR UV-vis absorbance and NIR reflectance data were collected for all experiments. The same chemical conditions and experimental protocol were used for all experiments. The process studied was the crystallization and dissolution of salicylic acid $\left(\mathrm{C}_{7} \mathrm{H}_{6} \mathrm{O}_{3}\right.$, molecular weight 138.12 , Fisher Scientific ACS certified, CAS 69-72-7) in a solvent mixture containing $52 \% \mathrm{w} / \mathrm{w}$ ethanol $\left(\mathrm{CH}_{3} \mathrm{CH}_{2} \mathrm{OH}\right.$, molecular weight 46.07, EMD 200 proof, CAS 64-17-5) and 48\% w/w deionized water.

Reactor and Instrument Setup. The small-scale batch experiments were performed in a custom jacketed $50 \mathrm{~mL}$ reaction vessel made in-house at East Carolina University. As shown in Figure 1, the reactor and lid were specifically designed

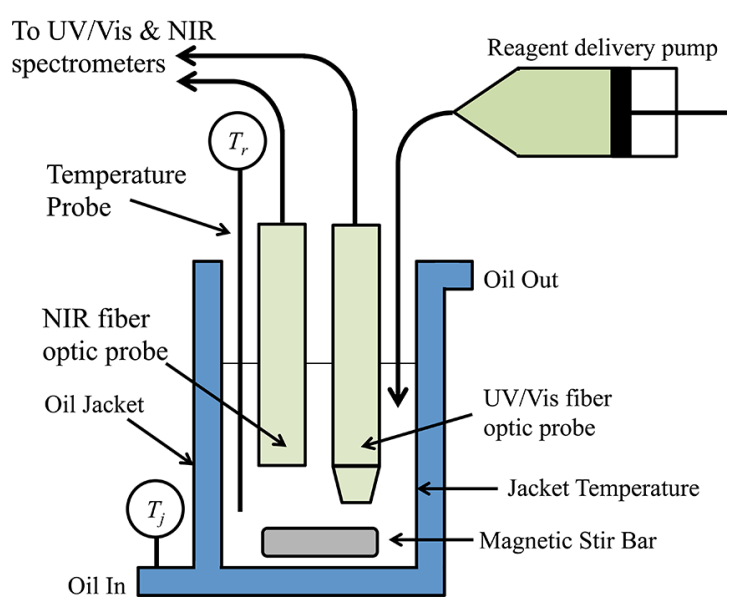

Figure 1. Schematic representation of the in-house small-scale semibatch reactor.

to accept an ATR UV-vis probe, a NIR reflectance probe, a temperature probe, and two PTFE feed lines. The reactor jacket was thermostated using a MGW Lauda RMS6 heater/ chiller, and the PTFE feed lines were connected to an automated syringe pump. The reactor temperature, $T_{r}$, jacket temperature, $T_{j}$, and reagent additions from the pump were monitored and controlled by WinISO software from H.E.L. Inc. (Lawrenceville, NJ). Stirring was controlled by an IKA Labortechnik RCT Basic magnetic stirrer and a PTFE-coated stir bar placed in the reaction vessel. Before an experiment was initiated, the jacket temperature was set to a precisely controlled constant value of $25{ }^{\circ} \mathrm{C}$. The reactor temperature was monitored using a calibrated hastalloy temperature probe.

Spectroscopic Instrument and Data Acquisition. ATR UV-vis absorbance and NIR reflectance spectra were collected in the reactor using similar acquisition parameters. UV-vis measurements were made using a tec5USA (Plainview, NY) multichannel spectrophotometer with a resolution of $3 \mathrm{~nm}$ in the 190-1100 $\mathrm{nm}$ region and an ATR fiber-optic probe (Hellma 661.821 model, $205 \mathrm{~mm}, 125 \mathrm{~mm}$ o.d., sapphire crystal). UV-vis absorbance spectra were measured at a rate of $1 \mathrm{spectrum} / \mathrm{s}$ and averaged at $30 \mathrm{~s}$ intervals using MultiSpec Pro Process software designed by tec5USA. For all experiments, dark current spectra were acquired before the start of each experiment, and an ethanol-water mixture was used to acquire a reference spectrum before each run.

NIR measurements were made using a FOSS NIRSystems (Silver Spring, MD) model 6500 scanning spectrophotometer with a resolution of $4 \mathrm{~nm}$ in the $1100-2500 \mathrm{~nm}$ region and a diffuse reflectance fiber-optic probe (FOSS, $1.5 \mathrm{~m}, 1 / 2$ in. o.d. $\times 12$ in., sapphire lens). NIR reflectance data were measured at a rate of 1 spectrum/s and averaged at $30 \mathrm{~s}$ intervals using Vision software designed by FOSS NIRSystems. A ceramic disk was used to measure a reference spectrum before each run.

Experimental Design. For each experiment, the reactor was initially charged with $20 \mathrm{~mL}$ of the solvent mixture (52\% 
ethanol, $48 \%$ water) and maintained at $25{ }^{\circ} \mathrm{C}$. In the first batch, four precisely measured aliquots of solid salicylic acid in the range of $0.7-0.9 \mathrm{~g}$ totaling $3.2 \mathrm{~g}$ were introduced into the reactor at $10 \mathrm{~min}$ intervals. A slurry of solid salicylic acid in equilibrium with a saturated solution was achieved after the fourth addition. In replicate batches, a single addition of $3.2 \mathrm{~g}$, precisely measured $( \pm 0.001 \mathrm{~g})$, of solid salicylic acid was introduced into the reactor to achieve a saturated solution in one step. The reactor temperature was then increased to $35^{\circ} \mathrm{C}$ in order to dissolve all the remaining solid material. Subsequently, the temperature was decreased to $25{ }^{\circ} \mathrm{C}$ to produce a metastable supersaturated solution. After $30 \mathrm{~min}$ the well-stirred supersaturated solution was seeded with an additional aliquot of $2.5 \mathrm{~g}$ of solid salicylic acid precisely measured $( \pm 0.001 \mathrm{~g})$, at which point crystallization occurred. After an equilibration time of $40 \mathrm{~min}$ an automated syringe pump was used to deliver aliquots of fresh solvent for the dissolution phase of the experiment. The dissolution study was conducted by introducing six aliquots of $4 \mathrm{~mL}$ of fresh solvent precisely measured $( \pm 0.02 \mathrm{~g})$, into the reactor at 20 or $40 \mathrm{~min}$ intervals. A more detailed description of the experimental batch protocol is provided in the Results and Discussion section. Collection of ATR UV-vis absorbance and NIR reflectance spectra was continued every $30 \mathrm{~s}$ during the dissolution phase of the experiment.

In an independent experiment, a well-stirred slurry was prepared in a graduated cylinder and allowed to equilibrate overnight at $25{ }^{\circ} \mathrm{C}$ using the same amounts of reagents described above. The volume of the resulting heterogeneous mixture was determined to be $22.7 \mathrm{~mL}$. After allowing the solid material to settle at $25{ }^{\circ} \mathrm{C}$ for several hours, the density of an aliquot of the saturated solution was determined to be $0.90 \mathrm{~g} /$ $\mathrm{mL}$ by use of a $10 \mathrm{~mL}$ pycnometer.

Kinetic Models for Slurries. Slurries used in reaction mixtures undergo competing processes of dissolution and precipitation. To model these processes, PBEs are often used to study these kinds of mechanisms. Crystal growth is usually described by thin-film theory, where a mass transfer process across the diffusion layer surrounding the crystal is in competition with solute deposition onto the surface of the crystal.

$$
\begin{aligned}
& r_{\mathrm{c}}(t)=\frac{\Phi_{\mathrm{s}} \mathrm{MW}_{s} k_{\mathrm{c}}^{\prime}}{3 d_{s} \Phi_{\mathrm{v}}} \eta_{\mathrm{r}}\left(c(t)-c_{\mathrm{sat}}\right)^{g} \\
& r_{\mathrm{d}}(t)=\frac{2 \mathrm{MW}_{s} k_{\mathrm{d}}^{\prime}}{d_{s}}\left(c_{\mathrm{sat}}-c(t)\right)
\end{aligned}
$$

In eqs 1 and $2, r_{\mathrm{c}}(t)$ and $r_{\mathrm{d}}(t)$ are the instantaneous rates of crystallization and dissolution, respectively, of solute $S$ at time $t$; $\eta_{\mathrm{r}}$ is the effective size factor introduced by Garside; ${ }^{24} k_{\mathrm{c}}^{\prime}$ and $k_{\mathrm{d}}^{\prime}$ are kinetic rate constants; $\mathrm{MW}_{s}$ is the molecular weight of $S$; $d_{s}$ is the density of $S$; $c_{\text {sat }}$ is the solubility of $S$; $c(t)$ is the bulk concentration of $S$ at time $t ; g$ is the rate order of crystal growth. The parameters $\Phi_{\mathrm{s}}$ and $\Phi_{\mathrm{v}}$ are surface and volumetric shape factors, respectively. (See Table1 for a list of symbols.)

In the present application of kinetic modeling, the solute $S$ was salicylic acid and the reactor content was assumed to be well-mixed. Volume changes were assumed to be additive, and the length, surface, and volumetric shape factors for the crystals were assumed to remain approximately constant throughout the duration of the experiment. It was also assumed that there was no agglomeration of crystal particles or breakage of large

\begin{tabular}{|c|c|c|c|}
\hline variable & dimension & description & units \\
\hline$a_{\mathrm{NIR}}$ & $(1 \times 1)$ & NIR reflectance coefficient & $1 / \mathrm{g}$ \\
\hline $\mathbf{a}_{\mathrm{UV}}$ & $(n w \times 1)$ & $\begin{array}{l}\text { ATR UV-vis pure component } \\
\text { spectrum of solute } S\end{array}$ & $\mathrm{~L} / \mathrm{mol}$ \\
\hline$c(t)$ & $(1 \times 1)$ & $\begin{array}{l}\text { concentration of solute } S \text { in liquid } \\
\text { bulk at time } t\end{array}$ & $\mathrm{~mol} / \mathrm{L}$ \\
\hline c & $(n t \times 1)$ & concentration of $S$ in liquid bulk & $\mathrm{mol} / \mathrm{L}$ \\
\hline $\mathrm{c}_{\mathrm{abs}}$ & $(n t \times 1)$ & concentration of absorbing solute $S$ & $\mathrm{~mol} / \mathrm{L}$ \\
\hline$c f$ & $(1 \times 1)$ & $\begin{array}{l}\text { correction factor for ATR UV-vis } \\
\text { surface enhancement }\end{array}$ & \\
\hline$c_{\text {in }}$ & $(1 \times 1)$ & concentration of solid solute $S$ & $\mathrm{~mol} / \mathrm{L}$ \\
\hline$c_{\text {sat }}$ & $(1 \times 1)$ & saturated concentration of solute $S$ & $\mathrm{~mol} / \mathrm{L}$ \\
\hline$c_{0}$ & $(1 \times 1)$ & initial concentration of solute $S$ & $\mathrm{~mol} / \mathrm{L}$ \\
\hline$d_{S}$ & $(1 \times 1)$ & density of solute $S$ & $\mathrm{~g} / \mathrm{L}$ \\
\hline$f(t)$ & $(1 \times 1)$ & flow-in rate of solvent at time $t$ & $\mathrm{~L} / \mathrm{min}$ \\
\hline$g$ & $(1 \times 1)$ & rate order of crystal growth & \\
\hline $\mathbf{H}$ & & $\begin{array}{l}\text { crystallization/dissolution model } \\
\text { (here eqs } 10-12 \text { ) }\end{array}$ & \\
\hline $\mathbf{I}_{n t}$ & $(n t \times n t)$ & identity matrix & \\
\hline$k_{\mathrm{c}}^{\prime}$ & $(1 \times 1)$ & rate constant of crystal growth & $\begin{array}{l}\mathrm{L}^{g-2} /\left(\mathrm{mol}^{g-2}\right. \\
\min )\end{array}$ \\
\hline$k_{\mathrm{c}}$ & $(1 \times 1)$ & $\begin{array}{l}\text { effective rate constant of crystal } \\
\text { growth }\end{array}$ & $\begin{array}{l}\mathrm{L}^{g-1} /\left(\mathrm{mol}^{g-1}\right. \\
\min )\end{array}$ \\
\hline$k_{\mathrm{d}}^{\prime}$ & $(1 \times 1)$ & rate constant of dissolution & $\mathrm{mol} /(\mathrm{L} \mathrm{min})$ \\
\hline$k_{\mathrm{d}}$ & $(1 \times 1)$ & effective rate constant of dissolution & $\begin{array}{l}\mathrm{L}^{n-1} /\left(\mathrm{mol}^{n-1}\right. \\
\min \mathrm{g})\end{array}$ \\
\hline$m(t)$ & $(1 \times 1)$ & undissolved mass of solute $S$ at time $t$ & $\mathrm{~g}$ \\
\hline $\mathbf{m}$ & $(n t \times 1)$ & undissolved mass of solute $S$ & g \\
\hline $\mathrm{MW}_{S}$ & $(1 \times 1)$ & molecular weight of solute $S$ & $\mathrm{~g} / \mathrm{mol}$ \\
\hline$m_{0}$ & $(1 \times 1)$ & initial undissolved mass of solute $S$ & g \\
\hline$n$ & $(1 \times 1)$ & rate order of dissolution & \\
\hline$n p$ & $(1 \times 1)$ & $\begin{array}{l}\text { number of adjustable model } \\
\text { parameters (here } n p=4)\end{array}$ & \\
\hline$n t$ & $(1 \times 1)$ & number of time points & \\
\hline$n w$ & $(1 \times 1)$ & number of UV-vis wavelengths & \\
\hline p & $(n p \times 1)$ & vector of adjustable model parameters & \\
\hline $\mathbf{p}^{\mathrm{L}}$ & $(n p \times 1)$ & lower limits of model parameters & \\
\hline $\mathbf{p}^{\mathrm{U}}$ & $(n p \times 1)$ & upper limits of model parameters & \\
\hline$r_{\mathrm{c}}(t)$ & $(1 \times 1)$ & rate of crystal growth at time $t$ & $\mathrm{~mol} /(\mathrm{L} \mathrm{min})$ \\
\hline$r_{\mathrm{d}}(t)$ & $(1 \times 1)$ & rate of dissolution at time $t$ & $\mathrm{~mol} /(\mathrm{L} \min )$ \\
\hline$r_{\text {in }}(t)$ & $(1 \times 1)$ & rate of seeding at time $t$ & $\mathrm{~mol} /(\mathrm{L} \min )$ \\
\hline $\mathbf{r}_{\mathrm{NIR}}$ & $(n t \times 1)$ & NIR reflectance residuals & \\
\hline $\mathbf{R}_{\mathrm{UV}}$ & $(n t \times n w)$ & ATR UV-vis absorbance residuals & \\
\hline$s$ & $(n t \times 1)$ & degree of supersaturation of solute $S$ & $\mathrm{~mol} / \mathrm{L}$ \\
\hline$S$ & & solute (here salicylic acid) & \\
\hline$s s q$ & $(1 \times 1)$ & sum of squared residuals & \\
\hline $\mathrm{T}$ & & operator of matrix transposition & \\
\hline$t$ & $(1 \times 1)$ & time instant & $\min$ \\
\hline$v(t)$ & $(1 \times 1)$ & liquid volume of the slurry at time $t$ & $\mathrm{~L}$ \\
\hline $\mathbf{v}$ & $(n t \times 1)$ & liquid volume of the slurry & $\mathrm{L}$ \\
\hline$v_{0}$ & $(1 \times 1)$ & initial liquid volume of slurry & $\mathrm{L}$ \\
\hline $\mathbf{y}_{\mathrm{NIR}}$ & $(n t \times 1)$ & NIR reflectance data & \\
\hline $\mathbf{Y}_{\mathrm{UV}}$ & $(n t \times n w)$ & ATR UV-vis absorbance spectra & \\
\hline$\eta_{\mathrm{r}}$ & $(1 \times 1)$ & effective size factor & \\
\hline$\Phi_{s}, \Phi_{\mathrm{v}}$ & $(1 \times 1)$ & surface and volumetric shape factors & \\
\hline+ & & Moore-Penrose pseudoinverse & \\
\hline
\end{tabular}

Table 1. List of Notations

crystals due to shear forces from mixing. Under these assumptions, eqs 1 and 2 were simplified by combining constants to provide eqs 3 and 4, respectively.

$$
\begin{aligned}
& r_{\mathrm{c}}(t)=k_{\mathrm{c}}\left(c(t)-c_{\text {sat }}\right)^{g} \\
& r_{\mathrm{d}}(t)=k_{\mathrm{d}} m(t)\left(c_{\text {sat }}-c(t)\right)^{n}
\end{aligned}
$$




$$
r_{\text {in }}(t)=\frac{f(t)}{v(t)} c_{\text {in }}
$$

Eq 3 describes the crystal growth process where $k_{\mathrm{c}}$ is an effective rate constant of crystal growth representing the accumulation of all parameters and constants in eq 1. Eq 4 describes the dissolution process, where $m(t)$ is the undissolved mass of salicylic acid at time $t, k_{\mathrm{d}}$ is an effective dissolution rate constant, and $n$ is a rate order for the dissolution. Introduction of $m(t)$ into eq 4 helped to improve the quality of fitting. Addition of this term has also the effect of forcing the rate of dissolution to zero as the mass of undissolved solute approaches zero and may help compensate for the fact that some of the simplifying assumptions may not be strictly met. Eq 5 describes the seeding step, where $r_{\text {in }}(t)$ is the seeding rate, $f(t)$ is the time-dependent volumetric flow-in rate of salicylic acid seed crystals, $v(t)$ is the liquid volume of the slurry at time $t$, and $c_{\text {in }}$ is the concentration of solid salicylic acid (here equal to the molar volume of the solid).

Beer's Law and Reflectance Relation. According to Beer's law, UV-vis absorbance data collected in the $(n t \times n w)$ dimensional matrix $\mathbf{Y}_{\mathrm{UV}}$ is the sum of the contributions of all absorbing species at $n w$ wavelengths and $n t$ time points. Singlewavelength NIR reflectance data was collected at $1100 \mathrm{~nm}$ in the $n t$-dimensional vector $\mathbf{y}_{\mathrm{NIR}}$. At $1100 \mathrm{~nm}$ absorption by salicylic acid and solvents in the slurry is negligible, thereby providing a univariate measure $\mathbf{y}_{\mathrm{NIR}}$ of light scattering from particles in the slurry, that is, free from the interference of absorption signals. As salicylic acid is the only light-absorbing and light-scattering species present in the system under investigation, linear relations between absorbance and concentration (Beer's law) and between reflectance and mass of undissolved solid (reflectance relation) can be defined in the following vector forms:

$$
\begin{aligned}
& \mathbf{Y}_{\mathrm{UV}}=\mathbf{c}_{\mathrm{abs}} \mathbf{a}_{\mathrm{UV}}^{\mathrm{T}}+\mathbf{R}_{\mathrm{UV}} \\
& \mathbf{y}_{\mathrm{NIR}}=\mathbf{m} a_{\mathrm{NIR}}+\mathbf{r}_{\mathrm{NIR}}
\end{aligned}
$$

where $c_{a b s}$ is the $n t$-dimensional vector of concentration of salicylic acid obtained by numerical integration of the differential equations specified in the kinetic model, $\mathbf{a}_{U V}$ denotes the $n w$-dimensional vector containing the ATR UVvis pure component spectrum (molar absorptivities) of salicylic acid, $\mathbf{m}$ represents the $n t$-dimensional vector of undissolved mass of solid salicylic acid, and $a_{\mathrm{NIR}}$ is a NIR reflectance coefficient. In the absence of UV-vis enhancement effects at the surface of the ATR crystal (described below), the concentration profile, $\mathbf{c}_{\mathrm{abs}}$, and the undissolved mass profile, $\mathbf{m}$, are obtained by numerical integration of the differential equations specified in the kinetic model. Nonlinear optimization of the model parameters is here used to fit the modeled absorbance and reflectance to the measured absorbance and reflectance data, respectively. A matrix of residuals $\mathbf{R}_{\mathrm{UV}}(n t \times$ $n w)$ and a vector of residuals $\mathbf{r}_{\mathrm{NIR}}(n t \times 1)$ are included in eqs 6 and 7 , respectively, to take into account the instrumental noise and the lack of fit.

Surface Enhancement Effect. In the present ATR UVvis measurements an enhancement of the absorbance signal in supersaturated conditions was observed. Other workers also have observed this phenomenon and attribute it to nucleation or weak adsorption on the surface of the ATR crystal, causing an increase in the absorbance at all wavelengths. ${ }^{45}$ The kinetic model used in these studies was adapted to incorporate an additional adjustable parameter used as a correction factor, $c f$, to model this phenomenon. The measured ATR UV-vis absorbance signal was systematically higher under supersaturated conditions than predicted by uncorrected model.

$$
\begin{aligned}
& s(t)= \begin{cases}c(t)-c_{\mathrm{sat}} & \text { if } c(t) \geq c_{\mathrm{sat}} \\
0 & \text { if } c(t)<c_{\mathrm{sat}}\end{cases} \\
& \mathbf{c}_{\mathrm{abs}}=\mathbf{c}+(c f) \mathbf{s}
\end{aligned}
$$

As previously introduced, $\mathbf{c}_{\mathrm{abs}}$ represents the concentration profile of absorbing salicylic acid in solution that is used in Beer's law (eq 6). This concentration vector is modified according to eq 9, using the modeled concentration profile $\mathrm{c}$ where $c(t)$ are the individual elements of $c$ resulting from the integration of the kinetic model (eq 10, below) and the degree of supersaturation $\mathbf{s}(n t \times 1)$ that results from applying eq 8 to all $n t$ time points.

Kinetic Model and Numerical Integration. Kinetic modeling starts by postulating a kinetic model, here named $\mathbf{H}$ and depending on eqs $3-5$. The kinetic model is generally based on elementary dynamic steps, in this case crystallization and dissolution processes. Kinetic model $\mathbf{H}$ is a system of ordinary differential equations (ODEs) given by eqs 10-12, which is numerically integrated and results in the concentration profile $\mathbf{c}(n t \times 1)$ and the undissolved mass profile $\mathbf{m}(n t \times 1)$ of salicylic acid; and the volume profile, $\mathbf{v}(n t \times 1)$, of the slurry.

$$
\begin{aligned}
& \frac{\mathrm{d} c}{\mathrm{~d} t}=-r_{\mathrm{c}}(t)+r_{\mathrm{d}}(t)-r_{\text {in }}(t) \\
& \frac{\mathrm{d} m}{\mathrm{~d} t}=\operatorname{MW}_{s} v(t)\left(r_{\mathrm{c}}(t)-r_{\mathrm{d}}(t)+r_{\text {in }}(t)\right) \\
& \frac{\mathrm{d} v}{\mathrm{~d} t}=f(t)-\frac{\mathrm{MW}_{s} v(t)}{d_{S}}\left(r_{\mathrm{c}}(t)-r_{\mathrm{d}}(t)\right)
\end{aligned}
$$

Eqs 9-12 depend on four adjustable parameters $\left(k_{\mathrm{d}}, k_{\mathcal{c}} n\right.$, and $c f)$ collected in a vector $\mathbf{p}$ of dimension 4; note that the rate order $g$ for the crystal growth is here treated as a fixed parameter. Eqs 10-12 depend on the liquid volume $v(t)$ of the slurry, the solvent volumetric flow-in rate $f(t)$, and on the dissolution, crystallization, and seeding rates $r_{\mathrm{d}}(t), r_{\mathrm{c}}(t)$, and $r_{\text {in }}(t)$, respectively. This system of ODEs is numerically integrated using initial conditions $c_{0}, m_{0}$, and $v_{0}$.

Elimination of the Linear Parameters. At each iteration of the nonlinear optimization, the pure component spectrum $\mathbf{a}_{\mathrm{UV}}$ for the ATR UV-vis measurements is linearly estimated in the region from 250 to $350 \mathrm{~nm}$ using the vector $\mathrm{c}_{\mathrm{abs}}$ and the matrix $\mathbf{Y}_{\mathrm{UV}}$, as shown in eq 13. Similarly, the linear coefficient $a_{\mathrm{NIR}}$ can also be extracted (see eq 14) from the NIR reflectance measurements at $1100 \mathrm{~nm}$, where a measure of pure light scattering is possible.

$$
\begin{aligned}
& \mathbf{a}_{\mathrm{UV}}^{\mathrm{T}}=\mathbf{c}_{\mathrm{abs}}^{+} \mathbf{Y}_{\mathrm{UV}} \\
& a_{\mathrm{NIR}}=\mathbf{m}^{+} \mathbf{y}_{\mathrm{NIR}}
\end{aligned}
$$

$\mathbf{c}_{\mathrm{abs}}^{+}$and $\mathbf{m}^{+}$are left pseudoinverse vectors, computed as $\mathbf{c}_{\mathrm{abs}}^{+}=$ $\left(\mathbf{c}_{\mathrm{abs}}^{\mathrm{T}} \mathbf{c}_{\mathrm{abs}}\right)^{-1} \mathbf{c}_{\mathrm{abs}}^{\mathrm{T}}$ and $\mathbf{m}^{+}=\left(\mathbf{m}^{\mathrm{T}} \mathbf{m}\right)^{-1} \mathbf{m}^{\mathrm{T}}$, respectively.

Nonlinear Optimization. The standard Newton-GaussLevenberg/Marquardt (NGL/M) algorithm was used to adjust the vector $\mathbf{p}$ of model parameters. The convergence of gradient methods such as NGL/M is superior to other methods, provided that the initial guesses for the parameters are close to 

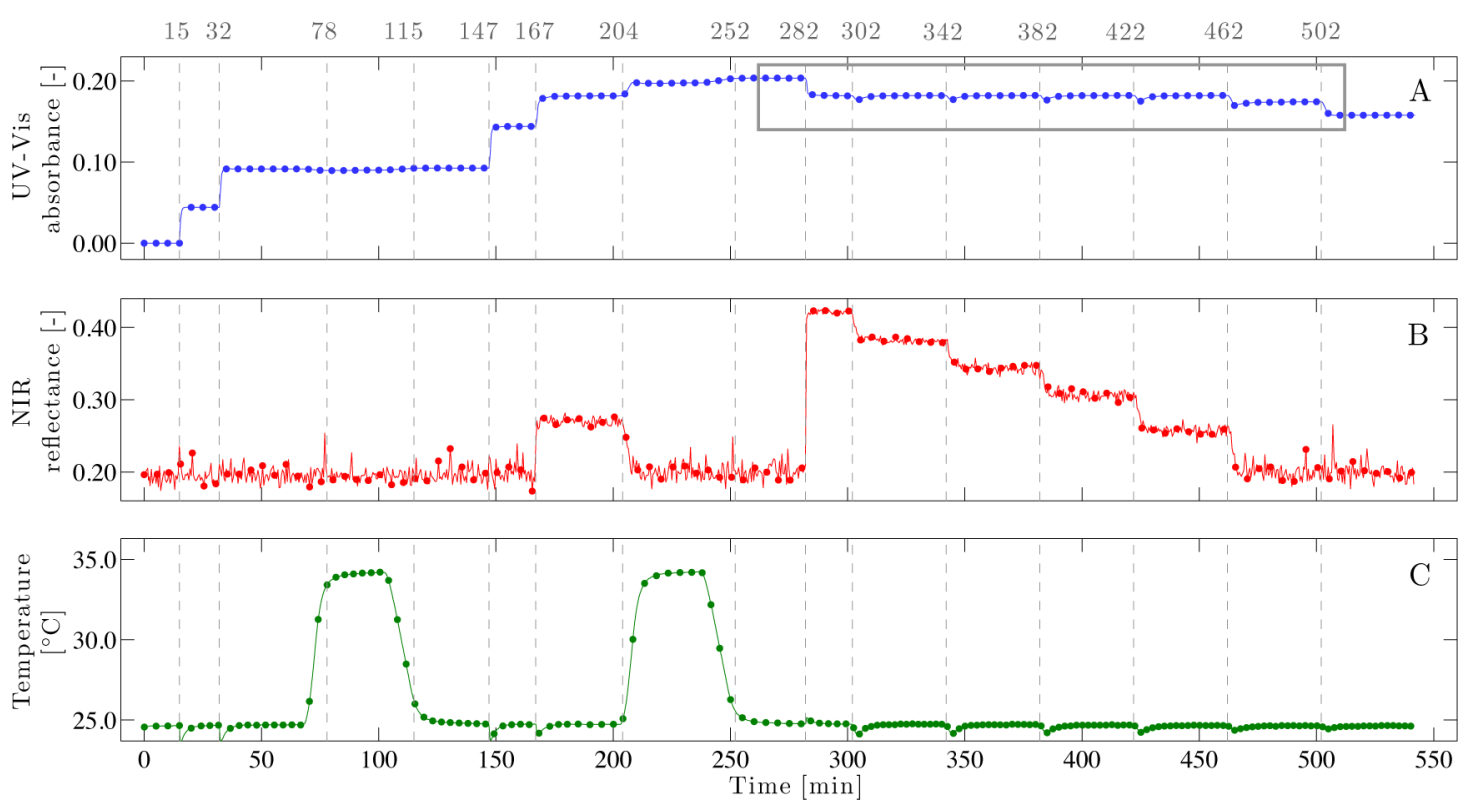

Figure 2. Comprehensive record of an experiment with (A) time-resolved ATR UV-vis absorbance data plotted at $307 \mathrm{~nm}$ but measured in the 260-370 $\mathrm{nm}$ region, (B) NIR reflectance data after preprocessing at $1100 \mathrm{~nm}$, and (C) temperature data.

their optimal values. ${ }^{46} \mathrm{NGL} / \mathrm{M}$ relies on the calculation of the derivative of the residuals with respect to each of the adjustable parameters. These derivatives were estimated by the technique of finite differences with a relative difference of $10^{-6}$. The convergence criterion was fixed to a relative change of $10^{-4}$ in the sum of squared residuals between successive iterations.

Lower and upper bounds for the adjustable parameters were implemented into $\mathrm{NGL} / \mathrm{M}$ as follows. If the values of parameters after applying the shifts computed by NGL/M exceeded maximum or minimum values, then the parameters were adjusted to upper or lower limits, respectively. Linear estimates of $\mathbf{a}_{\mathrm{UV}}$ (eq 13) and $a_{\mathrm{NIR}}$ (eq 14) are used to compute residuals $\mathbf{R}_{\mathrm{UV}}$ and $\mathbf{r}_{\mathrm{NIR}}$ from eqs 6 (Beer's law) and 7 (reflectance relation), respectively, as

$$
\begin{aligned}
& \mathbf{R}_{\mathrm{UV}}=\mathbf{Y}_{\mathrm{UV}}-\mathbf{c}_{\mathrm{abs}} \mathbf{a}_{\mathrm{UV}}^{\mathrm{T}}=\left(\mathbf{I}_{n t}-\mathbf{c}_{\mathrm{abs}} \mathbf{c}_{\mathrm{abs}}^{+}\right) \mathbf{Y}_{\mathrm{UV}} \\
& \mathbf{r}_{\mathrm{NIR}}=\mathbf{y}_{\mathrm{NIR}}-\mathbf{m} a_{\mathrm{NIR}}=\left(\mathbf{I}_{n t}-\mathbf{m m}^{+}\right) \mathbf{y}_{\mathrm{NIR}}
\end{aligned}
$$

where $\mathbf{I}_{n t}$ is the $n t$-dimensional identity matrix. The sum of squares, ssq, which is the driving force of the NGL/M optimization algorithm, is the sum of all squared residuals $\mathbf{R}_{\mathrm{UV}}$ and $\mathbf{r}_{\mathrm{NIR}}$.

$$
s s q=\sum_{i=1}^{n t} \sum_{j=1}^{n w} \mathbf{R}_{\mathrm{UV} i, j}{ }^{2}+\sum_{i=1}^{n t} \mathbf{r}_{\mathrm{NIR} i}{ }^{2}
$$

The least-squares optimization problem solved by NGL/M can be formulated as follows: minimize the sum of squared residuals by adjusting the values of a vector $\mathbf{p}$ of model parameters subjected to (s.t.) a postulated kinetic model $\mathbf{H}(\mathbf{p})$ of differential form and to upper and lower bounds $\mathbf{p}^{\mathrm{U}}$ and $\mathbf{p}^{\mathrm{L}}$, respectively. Mathematically, this problem can be formulated as

$$
\begin{array}{ll}
\min _{\mathbf{p}} & s s q \\
\text { s.t. } & \mathbf{H}(\mathbf{p}) \\
& \mathbf{p}^{L} \leq \mathbf{p} \leq \mathbf{p}^{U}
\end{array}
$$

\section{RESULTS AND DISCUSSION}

Figure 2 shows the entire record of an experiment, including ATR UV-vis absorbance at $307 \mathrm{~nm}$ (Figure 2A), NIR reflectance at $1100 \mathrm{~nm}$ (Figure $2 \mathrm{~B}$ ) and temperature (Figure 2C). The UV-vis absorbance data shown in Figure $2 \mathrm{~A}$ characterizes the solution phase and does not respond to solid material in the slurry. During the time interval from 15 to 167 min, four aliquots of solid salicylic acid were added, which gives a stepwise increase in the UV-vis absorbance profile of the liquid phase. These precisely measured amounts were used to confirm linear response of the ATR UV-vis probe under the concentration ranges used in these experiments. On the other hand it is well-known that changes in temperature produce small changes in the refractive index of liquid solvents which could affect the reliability of ATR measurements. In order to evaluate the significance of this effect, at $78 \mathrm{~min}$, the temperature of the solution was increased from 25 to $35^{\circ} \mathrm{C}$, which produced a corresponding $0.5 \%$ decrease in the UV-vis absorption at $307 \mathrm{~nm}$. This change in absorbance as a function of the temperature was considered negligible for the purpose of modeling.

At $115 \mathrm{~min}$, the temperature was reduced from $35^{\circ} \mathrm{C}$ back to $25^{\circ} \mathrm{C}$, and the absorbance returned to its original level. At this point, only a homogeneous solution was present in the reactor. At $167 \mathrm{~min}$, the last addition of salicylic acid produced a saturated solution. Only about $30 \%$ of the added material dissolved forming a slurry. At $204 \mathrm{~min}$, the temperature of the mixture was increased to $35{ }^{\circ} \mathrm{C}$, and the remaining solid salicylic acid dissolved producing a homogeneous solution. At $252 \mathrm{~min}$, the temperature of the mixture was cooled to $25^{\circ} \mathrm{C}$ producing a supersaturated solution. A corresponding significant increase in the absorption measured by the ATR UV-vis crystal was observed. This change was much larger $(+3.1 \%)$ than the effect of temperature $(-0.5 \%)$ observed at $78 \mathrm{~min}$. Experimental evidence suggested that the increase was most likely due to weak adsorption phenomena in the evanescence field near the ATR crystal surface. ${ }^{45}$ Crystallization on the surface of the ATR crystal can be ruled out because prior to the 


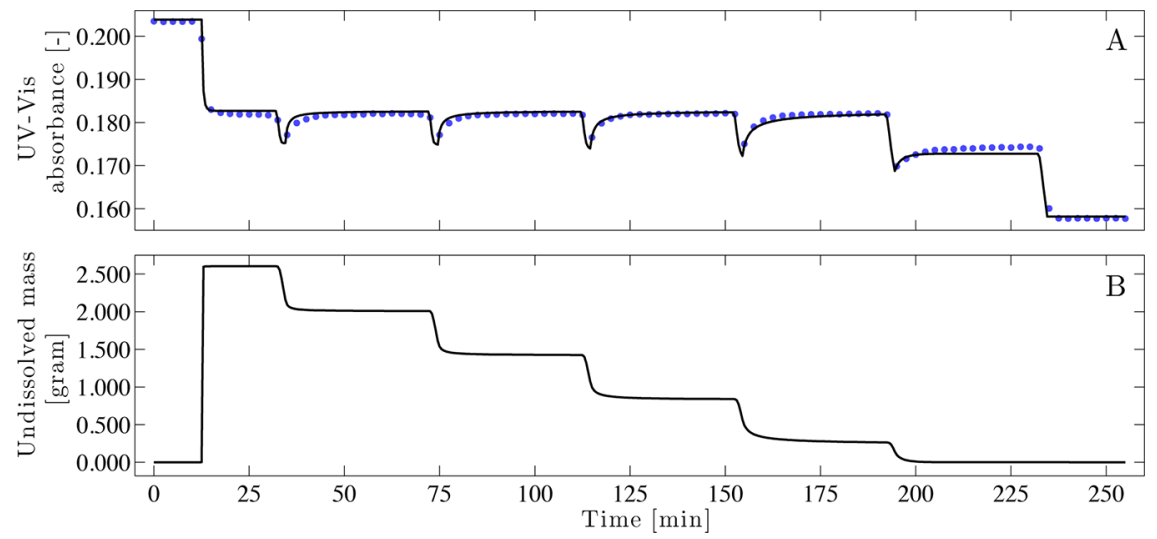

Figure 3. Expanded view of Figure $2 \mathrm{~A}$ between 262 and 512 min showing (A) measured (blue dots) and fitted (black line) ATR UV-vis absorbance data plotted at $307 \mathrm{~nm}$ but fitted in the $260-370 \mathrm{~nm}$ region and (B) modeled solid mass profile of salicylic acid. Time zero corresponds to 260 min in the experimental time. Seeded crystallization occurs at about $17 \mathrm{~min}$, and dissolution occurs at five intervals thereafter, corresponding to the programmed stepwise introduction of fresh solvent.
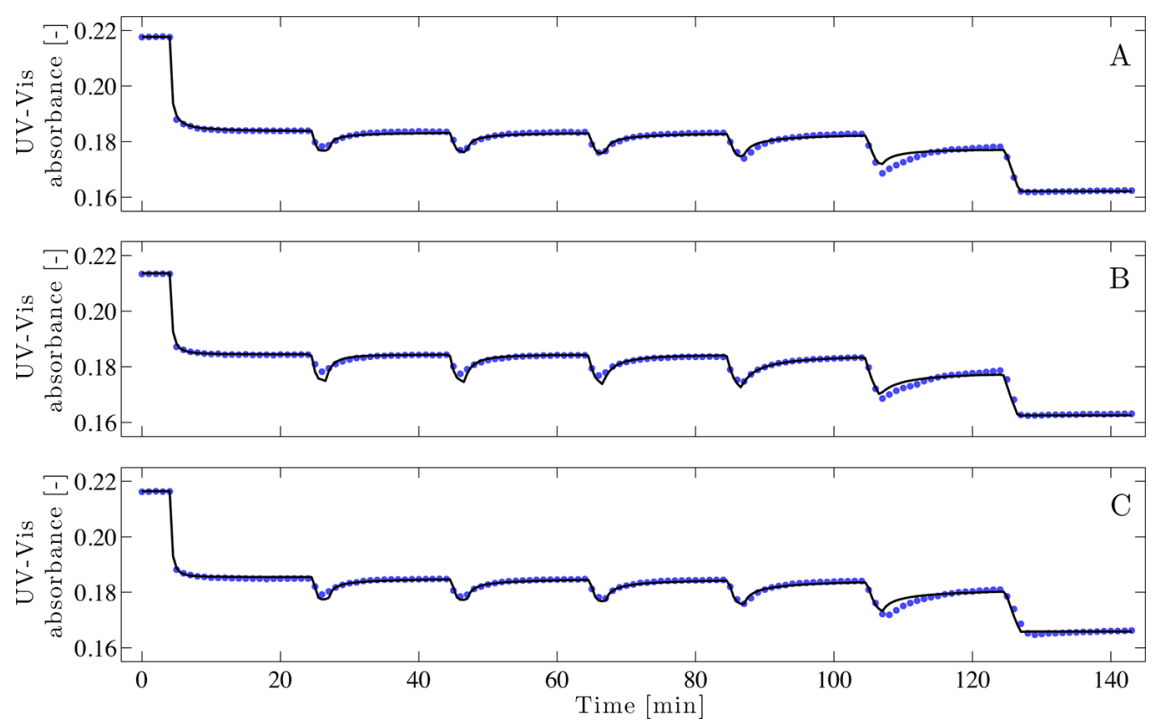

Figure 4. Time-resolved measured (blue dots) and fitted (black line) ATR UV-vis absorbance data plotted at $307 \mathrm{~nm}$ but fitted in the $260-370 \mathrm{~nm}$ region for three replicate experiments at $25{ }^{\circ} \mathrm{C}$. Time zero corresponds to $105 \mathrm{~min}$ in the experimental time.

quick cooling to $25^{\circ} \mathrm{C}$, the probe was submerged in a solution held at $35{ }^{\circ} \mathrm{C}$ for $30 \mathrm{~min}$. The resulting residual heat in the probe ensured that it stayed warmer than the surrounding solution, thus preventing crystallization of salicylic acid on the surface of the ATR crystal.

At $282 \mathrm{~min}, 2.5 \mathrm{~g}$ of solid salicylic was introduced at which point the supersaturated solution quickly crystallized to produce a saturated solution in equilibrium with the solid. At $302 \mathrm{~min}$, a $4 \mathrm{~mL}$ aliquot of fresh solvent was added, and a dissolution profile was observed, as shown in Figure 2A. Other additions of $4 \mathrm{~mL}$ aliquots of fresh mixture solvent were made at 342, 382, 422, 462, and $502 \mathrm{~min}$. After the fifth solvent addition at $462 \mathrm{~min}$, an undersaturated solution was produced (all salicylic acid dissolved). At $502 \mathrm{~min}$, an unsaturated solution was obtained and only dilution effects were observed.

Figure $2 \mathrm{~B}$ shows the NIR reflectance at $1100 \mathrm{~nm}$ indicating the presence of solid material from 167 to $204 \mathrm{~min}$ and from 282 to $462 \mathrm{~min}$. At $282 \mathrm{~min}$, when slurry was produced, a considerable increase in the NIR reflectance signal was observed. In Figure 2C, corresponding changes in the temperature can be observed. When solid salicylic acid at 25
${ }^{\circ} \mathrm{C}$ was introduced, a temporary decrease in temperature was observed as energy was absorbed by the dissolution process.

The top of Figure 3 shows an expanded view of Figure 2A between 262 and $512 \mathrm{~min}$. The blue-dotted line shows the ATR $\mathrm{UV}$-vis measurement at $307 \mathrm{~nm}$, and the black line shows the modeled UV-vis absorbance of dissolved salicylic acid obtained by fitting the ATR UV-vis. The bottom of Figure 3 shows the modeled amount of solid salicylic acid remaining in the slurry as a function of time. The results shown in Figure 3 were obtained by fitting a kinetic model to more than $511 \mathrm{UV}-$ vis spectra measured in the $260-370 \mathrm{~nm}$ region. Good fits were obtained for the spectral data. The overall standard deviation for the residuals of the UV-vis spectral data was $5 \times 10^{-4}$ absorbance units, slightly higher than the instrumental noise of $7 \times 10^{-5}$ absorbance units. Figure $3 \mathrm{~A}$ compares the fitted and measured UV-vis absorbance at $307 \mathrm{~nm}$, corresponding to the absorption peak maximum for the solute.

In order to evaluate the reproducibility of the experimental protocol and the model fitting process, three replicate experiments were performed with shorter time frames of operational conditions. This ensured that the model was robust 


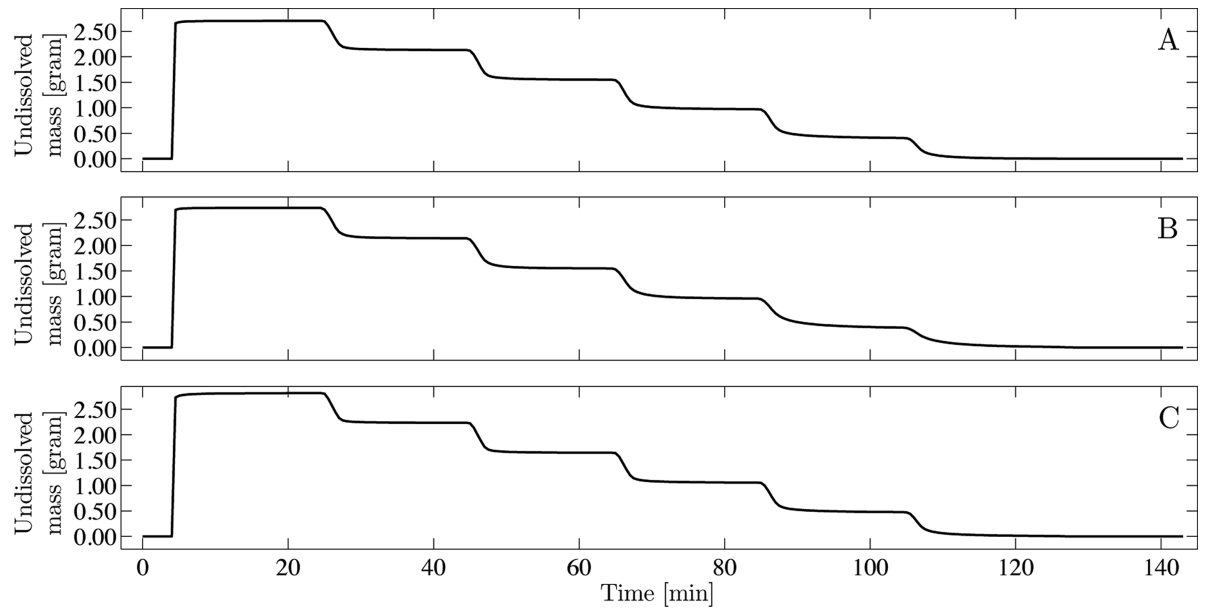

Figure 5. Estimated profiles of the undissolved mass of solid salicylic acid obtained by integration of eq 11 driven by the fitting of ATR UV-vis absorbance data for three replicate experiments. Time zero corresponds to $105 \mathrm{~min}$ in the experimental time.
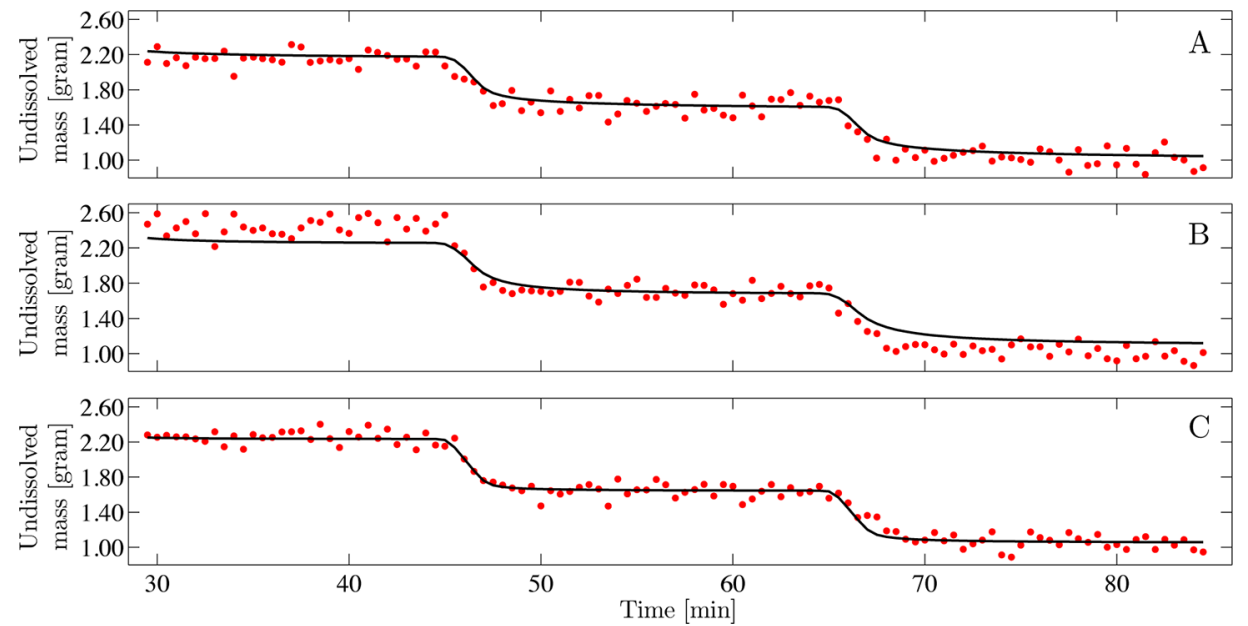

Figure 6. Modeled (black line) and PLS-estimated (red dots) undissolved mass profiles of salicylic acid obtained by fitting both ATR UV-vis multivariate absorbance data $(260-370 \mathrm{~nm})$ and NIR univariate reflectance data $(1100 \mathrm{~nm})$ for three replicate experiments.

with respect to changes in the experimental time frame. Three replicates were run at half of the time scale of the original experiment (i.e., fresh solvent was added at $20 \mathrm{~min}$ intervals rather than 40 min intervals).

In Figure 4, the ATR UV-vis absorbance profiles (liquid phase) of three replicate experiments are shown. The NIR reflectance measurements and temperature data are not shown but are comparable to these shown in Figure 2B and Figure 2C. Figure 4 shows good agreement between the fitted and measured UV-vis absorbance at $307 \mathrm{~nm}$, with slight variations most likely caused by small deviations from the assumptions made above (eqs 3-5). For example, in these experiments, slurries were assumed to be well-stirred, although this assumption does not strictly hold because small dead zones could be observed in the reactor behind the ATR UV-vis probe and the NIR reflectance probe. Another assumption was that the slurry was sufficiently well-mixed such that all particles of salicylic acid were suspended uniformly throughout the reactor vessel and that the stirring rate was relatively constant. Small changes in the stirring rate from batch to batch will show up as differences in the estimated rate constants for dissolution and crystallization. Last but not least, it was also assumed that the particle size distribution of the dissolving solid material remained approximately the same throughout the experiment.
For the purpose of this model fitting process, reasonably satisfactory fits were obtained within the limits of these assumptions.

Figure 5 shows the indirectly estimated profiles of undissolved salicylic acid for three replicate experiments, which result from the integration of kinetic model $\mathbf{H}$ (eqs 10-12) driven by the fitting of ATR UV-vis absorbance data. Note that NIR reflectance data were not used to produce these results. Indirect estimation of the solid mass is possible because the integrated dynamic model of eqs 10-12 enforces strict mass balance based on precisely specified initial conditions of each experiment.

Figure 6 shows the estimated solid mass profiles obtained by fitting both ATR UV-vis multivariate absorbance data (260$370 \mathrm{~nm})$ and NIR reflectance univariate data $(1100 \mathrm{~nm})$ compared to solid mass profiles estimated by partial least squares (PLS) for the second, third, and fourth dissolution steps of three replicate experiments. Note that PLS is used here for validation purposes only, that is, it is not used in the fitting process. The PLS calibration experiments were built as follows. First, a separate set of experiments were conducted to precisely determine the solubility of salicylic acid in the mixed solvent ( $52 \%$ ethanol, $48 \%$ water) at $25{ }^{\circ} \mathrm{C}$. Then, knowing the solubility limit, a total of eight calibration mixtures were 
Table 2. Comparison of Kinetic Modeling Results Using Only ATR UV-Vis Multivariate Absorbance Data (260-370 nm) or Using ATR UV-Vis Multivariate Absorbance Data Fused with NIR Univariate Reflectance Data $(1100 \mathrm{~nm})^{a}$

\begin{tabular}{|c|c|c|c|c|c|c|c|c|c|}
\hline & \multicolumn{2}{|c|}{ experiment 1} & \multicolumn{2}{|c|}{ experiment 2} & \multicolumn{2}{|c|}{ experiment 3} & \multirow[b]{2}{*}{ average } & \multirow[b]{2}{*}{$\mathrm{STD}^{b}$} & \multirow[b]{2}{*}{$\operatorname{RSD}^{c}$} \\
\hline & initial guess & optimal value & initial guess & optimal value & initial guess & optimal value & & & \\
\hline \multicolumn{10}{|c|}{ ATR UV-Vis Data Only } \\
\hline$k_{\mathrm{d}}^{d}$ & 79.41 & 67.90 & 59.43 & 35.38 & 109.9 & 55.84 & 53.04 & 16.44 & $31.0 \%$ \\
\hline$k_{\mathrm{c}}^{e}$ & 35.86 & 30.04 & 27.52 & 27.21 & 30.27 & 29.37 & 28.88 & 1.481 & $5.1 \%$ \\
\hline$n^{f}$ & 2.228 & 2.505 & 2.426 & 2.280 & 2.315 & 2.453 & 2.453 & 0.1181 & $4.9 \%$ \\
\hline \multicolumn{10}{|c|}{ ATR UV-Vis and NIR Reflectance Data } \\
\hline$k_{\mathrm{d}}{ }^{d}$ & 52.10 & 59.43 & 52.10 & 52.30 & 109.9 & 52.10 & 54.61 & 4.174 & $7.6 \%$ \\
\hline$k_{\mathrm{c}}^{e}$ & 28.78 & 27.52 & 28.78 & 32.92 & 30.27 & 28.78 & 29.74 & 2.821 & $9.5 \%$ \\
\hline$n^{f}$ & 2.576 & 2.426 & 2.576 & 2.789 & 2.315 & 2.576 & 2.597 & 0.1822 & $7.0 \%$ \\
\hline
\end{tabular}

${ }^{a}$ Rate order $g$ for the crystal growth was fixed to 1.5 , and saturation limit $c_{\text {sat }}$ was determined experimentally $(1.004 \mathrm{~mol} / \mathrm{L}) .{ }^{b}$ Standard deviation. ${ }^{c}$ Relative standard deviation. ${ }^{d}$ Rate constant of dissolution has units $\mathrm{L}^{n-1} /\left(\mathrm{mol}^{n-1} \mathrm{~min} \mathrm{~g}\right)$, with $n$ as the rate order of dissolution. ${ }^{e}$ Rate constant of crystal growth has units $\mathrm{L}^{g-1} /\left(\mathrm{mol}^{g-1} \mathrm{~min}\right)$, with $g$ as the rate order of crystal growth. ${ }^{f}$ Rate order $n$ for the dissolution has no units.

prepared from a well-stirred saturated solution of salicylic acid with known amounts of undissolved salicylic acid. The NIR reflectance spectra of these solutions were measured from 1100 to $1700 \mathrm{~nm}$. The PLS calibration model was constructed using these eight calibration mixtures in the range of 1100 to 1700 $\mathrm{nm}$ using three latent variables and MATLAB code written inhouse using the algorithm of Lorder et al. ${ }^{47}$ The resulting PLS calibration model was used to estimate the solid profiles shown in Figure 6 for comparison to the model estimated mass profiles. It should be emphasized that the reflectance $R$ was used in the PLS calibration rather than the conventional quantity $\log (1 / R)$, as the raw signal $R$ gave the best linearity with the fewest number of factors. On the basis of the results obtained from the PLS calibration, we elected to use reflectance, $R$, for our multivariate fitting process as opposed to $\log (1 / R)$.

The first and fifth dissolution steps were not included in Figure 6. The rate of dissolution for the first step was different from the remaining four which might have been due to fast dissolution of small/tiny particles present in the mixture of the solid phase. The fifth step is not shown because the amount of solid present in the reactor was too low to give a reliable NIR reflectance signal. It is well-known that NIR reflectance probes are not suitable for measuring systems with low light-scattering properties.

Table 2 shows the comparison between results of different procedures of kinetic modeling. The upper half of the table shows the results obtained by the fitting of ATR UV-vis measurements alone, and the bottom half of the table shows the results obtained by the combined fitting of ATR UV-vis absorbance and NIR reflectance measurements. The averaged fitting results from upper and lower portion of the table are quite similar to one another. The overall standard deviation for the residuals of the UV-vis spectral data in the three combined fitting batches ranged from $5 \times 10^{-4}$ to $7 \times 10^{-4}$ absorbance units, slightly higher than the expected fiber-optic ATR UV-vis measurement error of $7 \times 10^{-5}$ absorbance units, with 99.99299.996\% variance explained. These results were similar to the results obtained by fitting the UV-vis data alone. The overall standard deviation for the residuals of the NIR reflectance data ranged from 0.051 to 0.063 reflectance units with $93.8-96.3 \%$ variance explained. The overall standard deviation for the NIR residuals was slightly higher than the experimentally estimated measurement error of 0.039 reflectance units.

\section{CONCLUSION}

In this paper, an advanced measurement and modeling strategy was demonstrated to monitor the dissolution and crystallization of salicylic acid in a mixed solvent. The solid phase was monitored using in situ diffuse reflectance NIR spectroscopy, while the liquid phase was monitored using in situ ATR UVvis spectroscopy. In order to determine the kinetic parameters involved during the crystal growth and dissolution processes, a kinetic model describing these processes as a function of time was fitted to time-resolved multivariate spectral measurements. The suggested kinetic laws were sufficient in describing the set of four crystallization and dissolution experiments performed during this study. The parameters estimated in the model included dissolution and crystallization rate constants, dissolution rate order, as well as the degree of supersaturation and undersaturation that provides the driving force for crystallization and dissolution, respectively. In addition, this article has presented the methods to be used to carry out the fitting of a kinetic model to measured multivariate spectroscopic data of slurries (i.e., ATR UV-vis absorbance and NIR reflectance spectra): (i) postulation of the model and derivation of the differential equations, (ii) numerical integration of the model to yield the concentration profiles, and finally (iii) calculation of the pure component spectra and adjustment of models' rate constants to measured data. The model accurately estimated time-resolved profiles showing the concentration of solute in the liquid phase and the mass of solute in the solid phase. This enabled model estimation of the degree of supersaturation during conditions when crystallization took place and the degree of undersaturation during conditions when dissolution took place.

The goal of this research project was therefore achieved, that is, the development of a kinetic model that gives a statistically sufficient description of the dissolution/crystallization process for monitoring and control by multivariate spectroscopy.

\section{AUTHOR INFORMATION}

\section{Notes}

The authors declare no competing financial interest.

\section{ACKNOWLEDGMENTS}

This research was supported by the National Science Foundation (NSF) under Grant No. CHE-0750287, Grant Opportunities for Academic Liaison with Industry (GOALI), and the E.I. DuPont de Nemours and Co., Inc., Crop 
Protection Products and Engineering Technologies. Any opinions, findings, and conclusions or recommendations expressed in this material are those of the author(s) and do not necessarily reflect the views of the National Science Foundation.

\section{REFERENCES}

(1) Kourti, T. J. Chemom. 2003, 17, 93-109.

(2) Nomikos, P.; MacGregor, J. F. Chemom. Intell. Lab. Syst. 1995, 30, 97-108.

(3) Nomikos, P.; MacGregor, J. F. AIChE J. 1994, 40, 1361-1375.

(4) Wikstrom, C.; Albano, C.; Eriksson, L.; Friden, H.; Johansson, E.; Nordahl, A.; Rannar, S.; Sandberg, M.; Kettaneh-Wold, N.; Wold, S. Chemom. Intell. Lab. Syst. 1998, 42, 221-231.

(5) Wikstrom, C.; Albano, C.; Eriksson, L.; Friden, H.; Johansson, E.; Nordahl, A.; Rannar, S.; Sandberg, M.; Kettaneh-Wold, N.; Wold, S. Chemom. Intell. Lab. Syst. 1998, 42, 233-240.

(6) Wold, S.; Kettaneh, N.; Friden, H.; Holmberg, A. Chemom. Intell. Lab. Syst. 1998, 44, 331-340.

(7) Bugnon, P.; Chottard, J.-C.; Jestin, J.-L.; Jung, B.; Laurenczy, G.; Maeder, M.; Merbach, A. E.; Zuberbuehler, A. D. Anal. Chim. Acta 1994, 298, 193-201.

(8) Amrhein, M.; Srinivasan, B.; Bonvin, D.; Schumacher, M. M. Chemom. Intell. Lab. Syst. 1999, 46, 249-264.

(9) Molloy, K. J.; Maeder, M.; Schumacher, M. M. Chemom. Intell. Lab. Syst. 1999, 46, 221-230.

(10) Billeter, J.; Neuhold, Y. M.; Simon, L.; Puxty, G.; Hungerbühler,

K. Chemom. Intell. Lab. Syst. 2008, 93, 120-131.

(11) Ma, B.; Gemperline, P. J.; Cash, E.; Bosserman, M.; Comas, E. J. Chemom. 2003, 17, 470-479.

(12) Zogg, A.; Stoessel, F.; Fischer, U.; Hungerbuhler, K. Thermochim. Acta 2004, 419, 1-17.

(13) Puxty, G.; Maeder, M.; Rhinehart, R. R.; Alam, S.; Moore, S.; Gemperline, P. J. J. Chemom. 2005, 19, 329-340.

(14) Nilsson, P.; Puxty, G.; Wendt, O. F. Organometallics 2006, 25, $1285-1292$.

(15) Conway, W.; Wang, X. G.; Fernandes, D.; Burns, R.; Lawrance, G.; Puxty, G.; Maeder, M. J. Phys. Chem. A 2011, 115, 14340-14349.

(16) Puxty, G.; Maeder, M.; Radack, K. P.; Gemperline, P. J. Appl. Spectrosc. 2005, 59, 329-334.

(17) Zogg, A.; Fischer, U.; Hungerbuhler, K. Chemom. Intell. Lab. Syst. 2004, 71, 165-176.

(18) Gemperline, P.; Puxty, G.; Maeder, M.; Walker, D.; Tarczynski, F.; Bosserman, M. Anal. Chem. 2004, 76, 2575-2582.

(19) Billeter, J.; Srinivasan, S.; Bonvin, D. Anal. Chim. Acta. 2013 $767,21-34$

(20) Ehly, M.; Gemperline, P. J.; Nordon, A.; Littlejohn, D.; Basford, J. K.; De Cecco, M. Anal. Chim. Acta 2007, 595, 80-88.

(21) Maeder, M.; Neuhold, Y. M.; Puxty, G.; Gemperline, P. Chemom. Intell. Lab. Syst. 2006, 82, 75-82.

(22) Godany, T. A.; Neuhold, Y. M.; Hungerbuhler, K. Ind. Eng. Chem. Res. 2011, 50, 5982-5991.

(23) Randolph, A. D.; Larson, M. A. Theory of Particulate Processes: Analysis and Techniques of Continuous Crystallization; Academic Press: New York, 1971; p 260.

(24) Garside, J. Chem. Eng. Sci. 1985, 40, 3-26.

(25) Tavare, N. S. Sep. Purif. Methods 1993, 22, 93-210.

(26) Fevotte, G.; Klein, J. P. Chem. Eng. Sci. 1994, 49, 1323-1336.

(27) Fevotte, G.; Klein, J. P. Chem. Eng. J. Biochem. Eng. J. 1995, 59, $143-152$.

(28) Fevotte, G.; Klein, J. P. Chem. Eng. Res. Des. 1996, 74, 791-796.

(29) Fevotte, G.; Klein, J. P. Can. J. Chem. Eng. 1996, 74, 372-384.

(30) Monnier, O.; Fevotte, G.; Hoff, C.; Klein, J. P. Thermochim. Acta 1996, 289, 327-341.

(31) Monnier, O.; Fevotte, G.; Hoff, C.; Klein, J. P. Chem. Eng. Sci. 1997, 52, 1125-1139.

(32) Lewiner, F.; Klein, J. P.; Puel, F.; Fevotte, G. Chem. Eng. Sci. 2001, 56, 2069-2084.
(33) Dunuwila, D. D.; Carroll, L. B.; Berglund, K. A. J. Cryst. Growth 1994, 137, 561-568.

(34) Lewiner, F.; Fevotte, G.; Klein, J. P.; Puel, F. Ind. Eng. Chem. Res. 2002, 41, 1321-1328.

(35) Braatz, R. D.; Fujiwara, M.; Ma, D. L.; Togkalidou, T.; Tafti, D. K. Int. J. Mod. Phys. B 2002, 16, 346-353.

(36) Caillet, A.; Sheibat-Othman, N.; Fevotte, G. Cryst. Growth Des. 2007, 7, 2088-2095.

(37) Oullion, M.; Puel, F.; Fevotte, G.; Righini, S.; Carvin, P. Chem. Eng. Sci. 2007, 62, 833-845.

(38) Henry, M.; Puel, F. E.; Perrichon, P. D.; Wisniewski, C.; Fevotte, G. Int. J. Chem. React. Eng. [Online], 2008, 6. DOI: 10.2002/ 1542-6580 1670.http://www.degruyter.com/view/j/ijcre.2008.6.1/ ijcre.2008.6.1.1670/ijcre.2008.6.1.1670.xml?format=INT.

(39) Cornel, J.; Lindenberg, C.; Mazzotti, M. Cryst. Growth Des. 2009, 9, 243-252.

(40) Dokoumetzidis, A.; Macheras, P. Int. J. Pharm. (Amsterdam, Neth.) 2006, 321, 1-11.

(41) Verhaeghe, F.; Arnout, S.; Blanpain, B.; Wollants, P. Phys. Rev. E 2006, 73, 036316.

(42) Gao, J. Y. Anal. Chem. 2012, 84, 10671-10678.

(43) Cornel, J.; Mazzotti, M. Anal. Chem. 2008, 80, 9240-9249.

(44) Cornel, J.; Mazzotti, M. Ind. Eng. Chem. Res. 2009, 48, 1074010745 .

(45) Simon, L. L.; Nagy, Z. K.; Hungerbuhler, K. Chem. Eng. Sci. 2009, 64, 3344-3351.

(46) Puxty, G.; Maeder, M.; Hungerbuhler, K. Chemom. Intell. Lab. Syst. 2006, 81, 149-164.

(47) Lorber, A.; Wangen, L. E.; Kowalski, B. R. J. Chemom. 1987, 1, 19-31. 